\title{
Empowering Nurses, Midwives and Allied Health Professionals to Gain an Academic, Research and Quality Improvement Experience within Clinical Practice
}

\author{
*Cate Bell ${ }^{a b}$ \& Vivienne Colleran ${ }^{a}$ \\ a Western Sussex Hospitals NHS Foundation Trust, United Kingdom; balso funded by NIHR \\ 70@70 Senior Nurse \& Midwife Research Leaders Programme, United Kingdom.
}

\begin{abstract}
The NHS Multi-Professional Framework for Advanced Clinical Practice in England (Health Education England, 2017) sets out an expectation that all health and care professionals working at advanced clinical practice (ACP) level demonstrate a range of standard clinical academic capabilities across four pillars: clinical practice, leadership and management, education, and research. To achieve this, practitioners and employers need to work collaboratively to develop these capabilities and recognise how they contribute to improving the quality of care. However, the culture, leadership values and infrastructure of clinical practice environments limit opportunities for such clinical academic development (van Oostveen et al., 2017), resulting in a strong clinical bias amongst advanced practitioners with limited focus on research and leadership. To address this, we propose Nursing, Midwifery and Allied Health Professional (NMAHP) groups require specific development programmes that combine an academic, research and quality improvement experience grounded within clinical practice roles. In this article, we describe Western Sussex Hospitals NHS Foundation Trust Clinical Improvement Scholarship (CIS), a twelve-month programme combining work on a practice-based quality improvement project alongside education for leadership, research and quality improvement. Early indicators from the first and second cohorts suggest a significant increase in research and leadership knowledge with 'scholars' reporting increased commitment and confidence in utilising research evidence to improve care. Line managers also suggest a notable rise in professional confidence in the delivery of care and organisational competency. We therefore conclude that the CIS programme has impacted positively on professional capabilities and clinical quality within the Trust.
\end{abstract}

Keywords: advanced practice; clinical academic careers; leadership; multi-professional framework; nursing, midwifery and allied health professionals; NMAHP; professional confidence; professional development; quality improvement; research

*Corresponding Author: Dr Cate Bell, RM, RGN, DMid, MSc (Advanced Practice), MSc (Leadership), PgCHSCE. Head of Research/NMAHP Clinical Academic Programme Lead and NIHR $70 @ 70$ Nurse/Midwife Research Leader. Western Sussex Hospitals NHS Foundation Trust. UK. Email: Cate.Bell@wsht.nhs.uk

Journal URL: https://publications.coventry.ac.uk/index.php/pblh

Bell, C., \& Colleran, V. (2019). Empowering nurses, midwives and allied health professionals to gain an academic, research and quality improvement experience within clinical practice. International Journal of Practice-based Learning in Health and Social Care, 7(2), 69-79. DOI 10.18552/ijpblhsc.v7i2.641

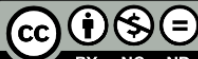

(C) 2019 Cate Bell \& Vivienne Colleran. This Open Access article is distributed under the terms of the Creative Commons Attribution Attribution-Non-Commercial No Derivatives 4.0 International License (https://creativecommons.org/licenses/by-nc-nd/4.0/ ), which permits unrestricted non-commercial use, distribution, and reproduction in any medium, provided the original work is properly cited and is unaltered. 


\section{Background}

The NHS Multi-Professional Framework for Advanced Clinical Practice in England (Health Education England, 2017) sets out an expectation that all health and care professionals working at advanced clinical practice level have standard capabilities across four key pillars and is defined as:

... a level of practice characterised by a master's level award or equivalent that encompasses the four pillars of clinical practice, leadership and management, education, and research, with demonstration of core capabilities and area specific clinical competence. (Health Education England, 2017, p. 8)

A key driver for this is the need for transformational change within the NHS workforce as described within the Long Term Plan (NHS England, 2019). Practitioners are challenged to innovate, develop healthcare, and to practise to their full potential to optimise their contribution to meeting patient and carer needs through new roles, models of service delivery and multi-professional working.

Advanced clinical practice has come into greater focus over recent years with regard to the potential for improving quality of care and leadership in the NHS (Anderson, 2018). Department of Health (DH) proposals to reform professional regulation in 2007 included the development of standards for higher levels of practice, and a position statement on Advanced Nursing Practice (ANP) in 2010 (Department of Health, 2010) provided a clear definition of the ANP role, including guidance on clinical practice, leadership, collaborative practice, quality improvement and developing self and others. These elements, with the addition of a specific requirement for capability in research, reflect the four pillars of advanced clinical practice capabilities set out in the NHS Multi-Professional Framework for Advanced Clinical Practice in England (Health Education England, 2017).

There has been some evaluation of how advanced practice roles impact healthcare quality. The evaluation by Miller et al. (2009) of the organisational impact of advanced clinical practitioner (ACP) roles, and others undertaken since (Begley et al., 2014; Elliott et al., 2016; Neville \& Swift, 2012), demonstrate a belief that advanced practitioners have improved service delivery, reduced length of stay, improved patient care and outcomes, reduced costs, increased efficiency and improved patient and staff satisfaction. In the majority of NHS trusts studied, advanced practitioners ran separate or parallel clinics to medical consultant colleagues and in some cases were managing their own caseloads. Advanced practitioners' roles are shown as having very tangible impacts, allowing the team to take on additional activities, reducing junior doctor hours and enabling medical practitioners to concentrate on complex cases. However, it is also highlighted that advanced practitioners lack research time (Begley et al., 2014), require improved skills in developing the evidence base to demonstrate their impact (Neville \& Swift, 2012) and frequently lack organisational support and opportunities to develop their leadership skills, limiting their effectiveness as change agents and innovators (Elliott et al., 2016).

Miller et al.(2009) also found that while the development of advanced practitioner roles in the trusts studied was largely led by a senior individual, typically a nursing director or consultant, often the posts were not embedded in strategic workforce plans, a feature which Hannay et al. (2013) suggest is critical for impact and sustainability. In the majority of sites, those taking on advanced practice roles were expected to have degree-level qualifications but there appeared to be few opportunities for advanced practitioners to engage in continuing professional development (CPD), particularly research (Begley et al., 2014) and leadership (Elliott et al., 2016). Whilst positive about their roles, those working as ACPs felt that opportunities for further career progression were limited with no clear career pathway other than moving into senior managerial positions. This would suggest that advanced clinical practitioners face challenges accessing development in relation to capabilities falling outside the clinical practice pillar which presents a significant barrier to the development of clinical academic practice (Baltruks \& Callaghan, 2018; Springett et al., 2014).

Development of an organisation's research environment is key in helping to deliver higher quality care and improved patient outcomes (Hanney et al., 2013; Jonker \& Fisher, 2018; NHS England, 2019). Developing research capabilities within the NHS workforce is therefore vital if we are to deliver the transformative and sustainable health and care services needed to meet future population needs $\underline{\text { NHS }}$ 
England, 2014, 2016, 2019). An understanding of the value of this would seem to be demonstrated through the inclusion of research as one of the four pillars of advanced clinical practice (Health Education England, 2017) and within the Key Lines of Enquiry (KLOE's) of the Care Quality Commission Inspection Framework (Care Quality Commission, 2018).

Currently, the development of clinical academics in the NHS is supported by the National Institute for Health Research (NIHR) (Health Education England/National Institute for Health Research, 2019; National Institute of Health Research, 2016). These roles are long established in the medical profession with a developing focus across nursing, midwifery and allied health professionals (NMAHPs) (CarrickSen et al., 2016; Strickland, 2017). However, as Westwood et al. (2018) highlight, although there is a national clinical academic training scheme in England focusing on the NMAHP workforce, it currently lacks the capacity to build the level of clinical academic leadership needed for the scale of innovation and improvements required across NHS health care.

Local initiatives are therefore needed to assist in building clinical academic capability and capacity in the workforce and to equip clinicians to work at an advanced practice level to improve care. The capabilities set out in the NHS Multi-professional Framework for Advanced Clinical Practice in England (Health Education England, 2017) are comprehensive, requiring significant focus on continuing professional development across all four pillars. To encourage the development of such capabilities across the clinical work force, organisations must be creative with opportunities for clinicians to develop their leadership and evidence-based practice/research skills alongside their clinical skills.

Clinicians developing their advanced practice/clinical academic capabilities also come from varied professional backgrounds, having different clinical experiences and different role expectations, meaning professional development pathways are individual. Professionals working at this level have a responsibility for their own continuing professional development; however, employing organisations need to ensure that there are appropriate opportunities to develop advanced practice capabilities with positive role models and mentors to support individuals' development. In order to develop a clinically focused workforce across the four pillars of advanced practice, flexible learning opportunities are required; delivery options may include clinical work-based units, formal specialist training modules, and interprofessional learning opportunities focused on areas such as leadership, and implementing and embedding change (Health Education England, 2017).

In this article, we describe Western Sussex Hospitals NHS Foundation Trust's (WSHFT) Clinical Improvement Scholarship (CIS) programme, a twelve-month programme for NMAHP's combining work on a practice-based quality improvement project alongside educational development in leadership, research, team working, change management and quality improvement (The King's Fund, 2017). The programme aims to increase professional confidence and to enhance professional development and personal growth in relation to the four pillars of advanced clinical practice capabilities.

\section{Method}

The WSHFT Clinical Academic Programme (CAP) was developed as a key feature of the Trust's Research and Innovation Strategy. The aim was to increase research engagement amongst nursing, midwifery and allied health professionals (NMAHP's) by offering opportunities to get involved at a range of levels combining clinical work with academic study and research. The CAP was informed by a staff engagement survey undertaken during development of the new Strategy with feedback highlighting a general lack of awareness regarding clinical academic roles and available opportunities to combine clinical and research careers, particularly within the nursing workforce. 


\begin{tabular}{|c|c|c|}
\hline $\begin{array}{l}\text { Advanced Clinical } \\
\text { Practice Pillar }\end{array}$ & $\begin{array}{l}\text { Key features of the WSHFT } \\
\text { Clinical Improvement Scholarship }\end{array}$ & What is involved \\
\hline Research & $\begin{array}{l}\text { Developing Evidence-Based Practice } \\
\text { (EBP) capabilities }\end{array}$ & $\begin{array}{l}\text { Complete accredited evidence base } \\
\text { practice module } \\
\text { Literature reviewing and synthesising } \\
\text { training with Clinical Librarians }\end{array}$ \\
\hline Education/Leadership & $\begin{array}{l}\text { Role modelling evidence-based } \\
\text { practice }\end{array}$ & $\begin{array}{l}\text { Initiate EBP supporting activities in } \\
\text { clinical setting e.g. journal clubs, } \\
\text { challenge of current practice using } \\
\text { evidence at improvement huddles. }\end{array}$ \\
\hline Research/Leadership & $\begin{array}{l}\text { Developing continuous improvement } \\
\text { capabilities } \\
\text { Evaluate own and multi-disciplinary } \\
\text { practice } \\
\text { Lead new practice and service } \\
\text { redesign }\end{array}$ & $\begin{array}{l}\text { Introduction to quality in the NHS } \\
\text { Accredited 'Yellow Belt' continuous } \\
\text { improvement training } \\
\text { Complete improvement project with } \\
\text { clinical team relating to Trust quality } \\
\text { priorities using 'lean' improvement } \\
\text { methodology }\end{array}$ \\
\hline Research & $\begin{array}{l}\text { Actively identify potential need for } \\
\text { further research to strengthen } \\
\text { evidence for best practice }\end{array}$ & $\begin{array}{l}\text { Complete a literature review in clinical } \\
\text { practice topic area }\end{array}$ \\
\hline Research & $\begin{array}{l}\text { Develop research methods } \\
\text { capabilities }\end{array}$ & $\begin{array}{l}\text { Research methods module } \\
\text { Develop research proposal }\end{array}$ \\
\hline Research/Leadership & $\begin{array}{l}\text { Facilitate collaborative links between } \\
\text { clinical practice and research }\end{array}$ & $\begin{array}{l}\text { Participate in collaborative research } \\
\text { groups }\end{array}$ \\
\hline Research & $\begin{array}{l}\text { Disseminate best practice research } \\
\text { findings }\end{array}$ & $\begin{array}{l}\text { Present literature review, and } \\
\text { improvement work to clinical audience } \\
\text { Complete accredited writing for } \\
\text { publication module } \\
\text { Write journal article for publication }\end{array}$ \\
\hline Education & $\begin{array}{l}\text { Critically assess and address own } \\
\text { learning needs }\end{array}$ & $\begin{array}{l}\text { Development of a personal } \\
\text { development plan with coaching }\end{array}$ \\
\hline Education & Engage in self-directed learning & $\begin{array}{l}\text { Self-directed learning supported by } \\
\text { peer action learning sets, coaching and } \\
\text { supervision. }\end{array}$ \\
\hline Leadership & Develop leadership capabilities & $\begin{array}{l}\text { Introduction to change management } \\
\text { and leadership in the NHS }\end{array}$ \\
\hline Education/leadership & $\begin{array}{l}\text { Support building the research culture } \\
\text { amongst NMAHPS }\end{array}$ & $\begin{array}{l}\text { Ambassadors role modelling of scope } \\
\text { and activities available for clinical } \\
\text { academic roles within NHS care to } \\
\text { colleagues }\end{array}$ \\
\hline
\end{tabular}

Table1: Key features of the WSHFT Clinical Improvement Scholarship 
The survey also highlighted that practitioners were often starting at very different levels - generally predegree with little previous experience of research or higher academic level study, and a few at postdoctoral level. The CAP was designed with a flexible format to offer a range of options to 'step-on' and off the programme at various academic levels and also to be complementary to other clinical academic routes for development, such as the NIHR Integrated Clinical Academic Programme (Health Education England/National Institute for Health Research, 2019).

The Clinical Improvement Scholarship (CIS) was conceived as a specific bridging programme within the wider CAP, recognising a need to support practitioners stepping up to formal educational opportunities at master's and doctoral level. It also recognised the importance of positioning research for practitioners as a continuum rather than as an absolute to offset low practitioner confidence and experience. The programme was developed in partnership with Health Education England Kent, Surrey and Sussex, and specifically designed to sit alongside the organisations' framework for quality improvement known as 'Patient First'. It was also strongly championed by senior NMAHP leaders within the organisation, including advanced clinical practitioners, with some already educated to doctoral level, including the Chief Nurse, and supported at Board level.

Clinical Improvement Scholarships aim to provide support at two levels, a Foundation Scholarship for those currently at pre-master's educational level, and a Senior Scholarship for those already at master's degree level. The new roles were advertised as an internal secondment for any NMAHP staff working within the Trust. All applicants were required to have some evidence of academic study at an appropriate level within the last five years and support from their line manager for the seconded time out of practice; staff within NHS Agenda for Change Bands 5-8a were eligible to apply. Successful candidates were supported with salary backfill for their clinical role to free them up for two days per week (15 hours) over 12 months with an expectation that they would work on a quality improvement project linked to the Trust's key quality areas alongside professional and personal research, leadership and educational development. The first cohort of scholars $(n=4)$ started in September 2017, a second cohort $(n=8)$ started in September 2018, each cohort representing a mixture of professional groups. Examples of improvement projects include:

\section{Reducing term admissions to the neonatal unit and improving experience}

This collaborative project led by a nurse CIS worked with neonatal and maternity care teams to improve clinical care and parents' experience by minimising mother/baby separation during this important period for bonding. The project resulted in development of a number of new care pathways including using evidence to introduce more effective approaches to managing and treating neonatal jaundice and neonatal skincare.

\section{Get up and get active: Supporting older patients with frailty to maximise function and maintain independence while in hospital}

This project led by an occupational therapist CIS involved working collaboratively with nursing, physiotherapy, dementia and volunteer teams to implement a training programme to encourage older patients to stay active whilst in hospital. This programme has already led to the introduction of specialist training in 'deconditioning' for new healthcare assistants in the Trust.

\footnotetext{
${ }^{1}$ Deconditioning is a complex process of physiological change following a period of inactivity, bedrest or sedentary lifestyle. It results in functional losses in such areas as mental status, degree of continence and ability to accomplish activities of daily living. (Gillis \& MacDonald, 2005, p. 17)
} 


\section{WSHFT Clinical Improvement Scholarship programme}

The Clinical Improvement Scholarship provides personalised development across the four pillars of Advanced Clinical Practice (ACP) with a specific focus on leadership and research capabilities; each scholar is required to develop a Personal Development Plan (PDP) during the first phase of the programme. The programme itself was developed by a local clinical academic project team, including the Chief Nurse, and includes a mixture of internal content taught by experienced local clinicians, externally accredited modules provided by higher education partners, self-directed learning, practical research or improvement project experience, participation in action learning sets, coaching and support within clinical practice. Academic supervision is provided for each scholar by a doctorally qualified member of the clinical academic team. The key features of the Clinical Improvement Scholarship Programme are shown in Table 1 , in relation to advanced clinical practice capabilities.

The leadership and management components are underpinned by the work of Kouzes and Posner (2012) on the five practices of exemplary leadership and is also linked with the NHS Leadership Academy Healthcare Leadership Model (NHS Leadership Academy, 2013). Scholars are asked to map the researcher development aspects of the programme against the VITAE Researcher Development Framework (VITAE, 2011).

The programme is not academically accredited in itself, a decision made to ensure that the wide range of learning and development needs can be tailored to individuals; however, all scholars are required to complete a quality improvement project and write an article for publication by the end of the year. A budget to enable completion of appropriate academic modules is made available for each scholar. Scholars identified the most useful development through their personal development plans supported by coaching, and senior scholars focused on post-master's educational offerings including preparation for doctoral-level study. Following development of close partnership with a local Higher Education Institute, the CIS programme can also be accredited at level 7 through the Accreditation of Prior Experiential Learning (APEL) route.

\section{Programme evaluation}

Cohorts 1 and 2 have now completed the full programme and all the scholars have become excellent evidence-based practice/research ambassadors engaging with and enthusing their local team members and the wider organisation. This has been evidenced by the widespread increase in interest in the programme, both from clinical practitioners and from line managers. The first cohort of the programme was evaluated informally through qualitative discussions with scholars based around completion of a self-report questionnaire, the 16-item EBP Beliefs Scale (Melnyk et al., 2008). Feedback from the scholars indicated that they observed an improvement in both their personal and professional confidence, particularly with regard to accessing and using research evidence to challenge and support change in practice. This is demonstrated through examples of the scholars' feedback below:

Never contemplated doctoral level study before but now, 9 months later have gained confidence and am keen to pursue. CIS Sept 2017

In practice there is too much firefighting - this gives you the opportunity to step outside of the day to day and think and reflect - this is what develops you the most. CIS Sept 2017

Has not just helped me but also others in the team around - lots more people asking questions and have asked what I am learning. CIS Sept 2017

In addition, the scholars' line managers were invited to comment during a one-to-one informal discussion of their perception of impact with regard to the scholar themselves and in the context of the wider team. The line manager's comments reflect a perception of growth in all the scholars' professional confidence and maturity, described as an observable change in scholar's ability to think more widely and to link their service goals and outcomes to wider trust objectives. Line managers also noted improved support for colleagues, engagement with research and evidence-based practice, and scholars' developing personal 
confidence to help challenge accepted practices. Scholars also demonstrated wider multi-disciplinary team (MDT) working, with increasing awareness and interest in research career opportunities including considering how new MDT roles might fit into traditional team structures.

\section{Discussion}

Evaluation of year one of the CIS programme demonstrates a positive impact, not only for the individual practitioners involved, but also more widely for patients and multi-professional staff groups across the organisation. Whilst the expectation during their twelve-month programme was to complete one clinical improvement project, all of the scholars successfully completed a number of improvement projects that demonstrate direct benefits for patients whilst also increasing their academic and research experience. Through the improvement projects, scholars have enhanced their multi-disciplinary team working and influenced the use of research evidence to inform everyday clinical care. They also report growth in confidence, both personally and professionally, particularly with regard to disseminating best practice through publication and conference presentations, with many of the scholars having more than one article, poster presentation or conference abstract accepted.

The wider impact of the programme also demonstrates how such roles can act to enthuse and motivate not just the practitioner concerned but also the wider healthcare team. As the line manager feedback highlights, the scholars have acted as catalysts within clinical teams to help support them to challenge existing practice using research evidence and leading to several examples of changes that have not only benefited patient care but, in many cases, also provided efficiencies of time and finance for the clinical service. The Clinical Improvement Scholar role offers an opportunity to combine development of practitioners' advanced clinical practice and clinical academic capabilities and is a potential alternative route for developing greater research capacity within the NMAHP workforce. In addition, it may offer an alternative option for other UK healthcare organisations where there is currently insufficient local structure to support the more academically driven models which rely on investment from a close Higher Education Institute (HEI) partner organisation.

\section{Enablers for success}

One of the key factors enabling the success of the CIS programme was the level of organisational readiness (Carrick-Sen et al., 2016). Indicators of organisational readiness include Trust Executive Board support and the embedding of clinical academic career development within the Trust's Research and Innovation Strategy. A further enabler for success of the programme to date may be the influence of having doctoral qualified nursing and midwifery programme champions within the Trust workforce, including the Director of Nursing. Clavelle et al. (2012) credit doctoral-level qualification with significantly increasing the transformational leadership practices of senior nurses, who are more likely to place value in academic development of clinical practitioners. Our experience suggests that provision of a bespoke programme, including mentoring and supervision from doctoral-prepared and clinically based supervisors, alongside more formal opportunities for academic educational development through HEI accredited modules, provides clinical academic role models in practice to help bridge the gap between academia and practice where existing, more formalised clinical academic roles do not currently exist.

In addition, the programme at Western may have also benefitted from the Trust's embedded clinical improvement culture known as 'Patient First' and in line with the Improving Care through Evidence (ICE) model for development of a more widespread research culture and increased research capacity described by Carrick-Sen et al. (2016). The Clinical Improvement Scholarship programme fits within the ICE model at the research awareness level, with the aim of facilitating clinical practitioners to move towards active research and research leadership levels.

\section{Challenges and barriers}

Miskelly and Duncan's (2014) evaluation of their local leadership programme suggests that having the time and space for reflection and self-assessment of professional and personal development needs was a critical feature of practitioners' development. This is also credited as a major strength of the Clinical 
Improvement Scholarship programme. While the provision of 'time out' to think and reflect was considered essential, it is striking how many of the scholars struggled with this type of activity, especially in the early stages of the programme. This perhaps demonstrates a culture which still places value principally on the 'doing' rather than 'thinking' aspects of professional practice (Springett et al., 2014).

Such a culture presents a barrier to the development of advanced clinical practitioner (ACP) capabilities and the embedding of more widespread clinical academic roles for healthcare practitioners. There is currently still significant emphasis on the service delivery aspects of ACP roles (van Oostveen et al., 2017) and there is often a tension between this and the availability of the required time out of practice for those undertaking post-graduate academic education (Springett et al., 2014). The CIS model aims to effect change amongst NMAHP groups through a process of role modelling and challenge embedded in practice, which demonstrate the positive effects for patients, clinical teams and services gained by supporting clinical academic roles and development of the ACP capabilities.

Lack of capacity for release of Scholars' time from clinical practice, despite available funding for replacement, was also a challenge encountered by the Clinical Improvement Scholarship programme. Given the current limitations related to staffing fulfilment, particularly within nursing and midwifery, this is likely to present similar challenges across other models. For those who do manage post-graduate education, a significant number of those with research experience gained through higher degrees no longer undertake any research-related activity once back in the clinical environment (van Oostveen, et al. 2017). Therefore, inadequate provision of time for research is a limiting factor for NMAHPs interested in pursuing research-related careers within the NHS (Baltruks \& Callaghan, 2018), as well as to the improvement of clinical care through direct translation of research to practice.

The design of the CIS programme seems to have slightly offset the time-release issue for some. In the manager's feedback it was suggested that this was related to the programme flexibility and to the clinical improvement focus. Integration of a practical service-focused and clinically based quality improvement project provided a tangible benefit to the clinical teams and maintained visibility of the scholar. This encouraged clinical managers and teams to offset the short-term effects of supporting time out of clinical practice for these types of roles with an observable benefit to the clinical environment and to the wider clinical practice team.

As highlighted in the NHS Multi-professional Framework for Advanced Clinical Practice in England (Health Education England, 2017), practitioners come from a range of clinical backgrounds and with a range of clinical and academic experience. In order to support their clinical academic development, the CIS programme is tailored to focus on developing the ACP capabilities most relevant to the current and future needs of each individual practitioner. This is achieved through personal coaching and supervision, alongside peer support through regular action learning sets and a flexible approach to supporting development and implementation of their quality-improvement projects. Throughout the process scholars gain academic, leadership, quality improvement, change management, teaching and presentation experience.

\section{Conclusion}

Recent literature shows that there are already several successful programme models which have been developed between NHS and HEI organisations intended to support integration of more NMAHP clinical academic roles to expand research capacity in NHS practice (Westwood et al., 2018), including the NIHR Integrated Clinical Academic programme Programme (Health Education England/National Institute for Health Research, 2019). However, these are typically hosted and situated within larger university hospital organisations and, while linked to practice, are primarily focused on an academic research model (Strickland, 2017). Where practice- and quality-improvement focused models similar to the Clinical Improvement Scholarship programme model do exist, such as the Chief Nurse Excellence in Care Junior Fellowship described by Bramley et al. (2018), these may be offered for singular professional groups or pay grades. The Clinical Improvement Scholarship programme therefore differs in offering support across a wide range of NMAHP groups and pay bands (Agenda for Change bands 5-8a) with development of 
capabilities across the four ACP pillars and a clinical quality-improvement focus within a District General Hospital environment.

The second cohort of eight NMAHPs began the programme in September 2018 and a more formal, externally led evaluation has been undertaken to consider how the CIS impacts on practitioners' personal and professional confidence and more directly on clinical practice. Plans have also been developed to widen organisational participation through collaboration with a partner NHS Trust joining the CIS programme as part of cohort 3 in September 2019. Local engagement with research has grown, with observable change in the organisational culture demonstrated by the increased number of applications for years 2 and 3 of the programme particularly amongst nursing and midwifery groups who have previously been under-represented. There is growing support from clinical managers and a rise in the numbers of NMAHPs exploring options for clinical academic development. While ACP clinical practice capabilities remain a central feature of ACP development, the main advantage of the CIS programme is in facilitating development of the research, education and leadership capabilities of NMAHPs to balance their strong clinical practice foundation.

Whilst small in scale, with only four individuals in the first cohort and eight in the second, the positive impact of the CIS role is evidenced by an increasing level of interest in this specific type of clinical/academic opportunity, embedded within clinical care and linked with the advanced clinical practice framework. The format of the programme has attracted interest from other practitioners and organisations at local, regional and national level, and the strengths of the CIS programme come from linking research, leadership and continuous improvement with the ACP four pillars. Therefore, the CIS programme potentially offers a new model through which research capacity could be increased within the existing NHS workforce and demonstrates the benefit of increasing practitioners' confidence in translating and embedding research evidence, to impact directly on patient care.

Early indicators from the first and second cohorts of scholars suggest a significant increase in research and leadership knowledge and skills. Observations and feedback from line managers and colleagues in practice also suggest a notable rise in professional confidence in the delivery of care and organisational competency. An increase in the scholars' professional confidence is also evidenced by their success in publication, conference presentation, professional networking and promotion into new advanced practice roles. Scholars report increased commitment and confidence in acting to improve care and to linking research and practice within their clinical roles, with all of them remaining within the organisation. We therefore conclude that the Clinical Improvement Scholarship Programme has positively impacted on scholars' experiences and professional capabilities, demonstrating their impact on clinical quality improvement to benefit patients, colleagues, and use of resources (NHS England, 2016) in the Trust.

\section{Acknowledgements}

Cate Bell is a National Institute of Health Research (NIHR) Senior Nurse and Midwife Research Leader. The views expressed in this article are those of the author and not necessarily those of the NIHR or the Department of Health and Social Care.

\section{ORCID}

Cate Bell: $\quad$ https://orcid.org/0000-0002-2175-7606

\section{References}

Anderson, C. (2018). Exploring the role of advanced nurse practitioners in leadership. Nursing Standard, 33(2), 29-33. https://doi.org/10.7748/ns.2018.e11044

Baltruks, D., \& Callaghan, P. (2018). Nursing, midwifery and allied health clinical academic careers in the UK. https://councilofdeans.org.uk/wp-content/uploads/2018/08/Nursing-midwifery-andallied-health-clinical-academic-research-careers-in-the-UK.pdf 
Begley, C., Murphy, K., Higgins, A., \& Cooney, A. (2014). Policy-makers' views on impact of specialist and advanced practitioner roles in Ireland: the SCAPE study. Journal of Nursing Management, 22(4), 410-422. https://doi.org/10.1111/jonm.12018

Bramley, L., Manning, J. C., \& Cooper, J. (2018). Engaging and developing frontline clinical nurses to drive care excellence: Evaluating the Chief Nurse Excellence in Care Junior Fellowship initiative. Journal of Research in Nursing, 23(8), 678-689. https://doi.org/10.1177/1744987118808843

Care Quality Commission. (2018). Key lines of enquiry for healthcare services. https://www.cqc.org.uk/guidance-providers/healthcare/key-lines-enquiry-healthcare-services

Carrick-Sen, D., Richardson, A., Moore, A., \& Dolan, S. (2016). Transforming healthcare through clinical academic roles in nursing, midwifery and allied health professions: A practical resource for healthcare provider organisations. https://councilofdeans.org.uk/wpcontent/uploads/2019/02/AUKUH-Transforming-Healthcare.pdf

Clavelle, J., Drenkard, K., Tullai-McGuinness, S., \& Fitzpatrick, J. (2012). Transformational leadership practices of chief nursing officers in Magnet organisations. Journal of Nursing Administration, 42(4), 195-201. https://doi.org/10.1097/NNA.0b013e31824ccd7b

Department of Health. (2010). Advanced Level Nursing: A position statement. https://www.gov.uk/government/publications/advanced-level-nursing-a-position-statement

Elliott, N., Begley, C., Sheaf, G., \& Higgins, A. (2016). Barriers and enablers to advanced practitioners' ability to enact their leadership role: A scoping review. International Journal of Nursing Studies. 60, 24-45. https://doi.org/10.1016/j.ijnurstu.2016.03.001

Gillis, A., \& MacDonald, B. (2005). Deconditioning in the Hospitalized Elderly. The Canadian Nurse, 101(6), 16-20.

Hanney, S., Boaz, A., Jones, T., \& Soper, B. (2013). Engagement in research: An innovative three-stage review of the benefits for health-care performance. Health Services and Delivery Research, 1(8). https://doi.org/10.3310/hsdr01080

Health Education England. (2017). Multi-professional framework for advanced clinical practice in England. https://www.hee.nhs.uk/sites/default/files/documents/Multiprofessional $\% 20$ framework $\% 20$ for $\% 20$ advanced $\% 20$ clinical $\% 20$ practice $\% 20 \mathrm{in} \% 20$ England.pdf

Health Education England (HEE)/National Institute for Health Research (NIHR). (2019). HEE/NIHR Integrated Clinical Academic Programme. https://www.nihr.ac.uk/our-researchcommunity/NIHR-academy/nihr-training-programmes/nihr-hee-ica-programme/

Jonker, L., \& Fisher, S. J. (2018). The correlation between National Health Service trusts' clinical trial activity and both mortality rates and care quality commission ratings: A retrospective crosssectional study. Public Health, 157, 1-6. https://doi.org/10.1016/j.puhe.2017.12.022

Kouzes, J., \& Posner, B. (2012). The Leadership Challenge: How to make extraordinary things happen in organizations (5th ed.). John Wiley and Sons.

Melnyk, B. M., Fineout-Overholt, E., \& Mays, M. (2008). The evidence-based practice beliefs and implementation scales: Psychometric properties of two new instruments. Worldviews on Evidence-Based Nursing, 5(4), 208-216. https://doi.org/10.1111/j.1741-6787.2008.00126.x 
Miller, L., Cox, A., \& Williams, J. (2009). Evaluation of the Advanced Practitioner Role. https://www.employment-studies.co.uk/system/files/resources/files/465.pdf

Miskelly, P., \& Duncan, L. (2014). 'I'm actually being the grown-up now': Leadership, maturity and professional identity development. Journal of Nursing Management, 22(1), 38-48. https://doi.org/10.1111/jonm.12030

National Institute of Health Research. (2016). Building a research career handbook. https://www.nihr.ac.uk/documents/building-a-research-career/20571

Neville, L., \& Swift, J. (2012). Measuring the impact of the advanced practitioner role: A practical approach. Journal of Nursing Management, 20(3), 382-389. https://doi.org/10.1111/j.13652834.2012.01356.x

NHS England. (2014). The five year forward view. https://www.england.nhs.uk/five-year-forward-view/

NHS England. (2016). Leading Change - Adding Value. A framework for nursing, midwifery and care staff. https://www.england.nhs.uk/wp-content/uploads/2016/05/nursing-framework.pdf

NHS England. (2019). NHS long term plan. http://www.longtermplan.nhs.uk

NHS Leadership Academy. (2013). The Healthcare Leadership Model: The nine dimensions of leadership behaviour. https://www.leadershipacademy.nhs.uk/wpcontent/uploads/2014/10/NHSLeadership-LeadershipModel-colour.pdf

Springett, K., Norton, C., Louth, S., Deaton, C., \& Young, A. (2014). Eliminate tensions to make research work on the front line. HSJ. https://www.hsj.co.uk/leadership/eliminate-tensions-tomake-research-work-on-the-front-

line/5075013.article?blocktitle=ResourceCentre\&contentID=8630\#.VDgAEfldVI5

Strickland, K. (2017). Developing an infrastructure to support clinical academic careers. British Journal of Nursing, 26(22), 1249-1252. https://doi.org/10.12968/bjon.2017.26.22.1249

The King's Fund. (2017). Embedding a culture of quality improvement. https://www.kingsfund.org.uk/publications/embedding-culture-quality-improvement

van Oostveen, C. J., Goedhart, N. S., Francke, A. L., \& Vermeulen, H. (2017). Combining clinical practice and academic work in nursing: A qualitative study about perceived importance, facilitators and barriers regarding clinical academic careers for nurses in university hospitals. Journal of Clinical Nursing, 26(23-24), 4973-4984. https://doi.org/10.1111/jocn.13996

Vitae. (2011). About the Vitae Researcher Development Framework. https://www.vitae.ac.uk/researchersprofessional-development/about-the-vitae-researcher-development-framework/about-the-vitaeresearcher-development-framework

Westwood, G., Richardson, A., Latter, S., Macleod Clark, J., \& Fader, M. (2018). Building clinical academic leadership capacity: Sustainability through partnership. Journal of Research in Nursing, 23(4), 346-357. https://doi.org/10.1177/1744987117748348 